\title{
Antioxidant properties of lipote (Syzygium polycephaloides (C.B. Rob.) Merr.) flesh and seeds as affected by maturity and processing method
}

\author{
${ }^{1}$ Ilano, M.C.R., ${ }^{1}$ Sartagoda, K.J.D., ${ }^{1}$ Flandez, L.E.L., ${ }^{1,2}$ Compendio, M.C.M., \\ ${ }^{1}$ Morales, D.B. and ${ }^{1, *}$ Castillo-Israel, K.A.T. \\ ${ }^{1}$ Institute of Food Science and Technology, College of Agriculture and Food Science, University of the \\ Philippines - Los Baños, Laguna, Philippines 4031 \\ ${ }^{2}$ School of Technology, University of the Philippines Visayas, Miagao, Iloilo, Philippines 5023
}

\begin{abstract}
Article history:
Received: 2 October 2020 Received in revised form: 18 November 2020

Accepted: 18 January 2021 Available Online: 29 April 2021
\end{abstract}

\section{Keywords:}

Lipote,

Antioxidants,

DPPH,

ABTS,

FRAP

DOI:

https://doi.org/10.26656/fr.2017.5(2).558

\begin{abstract}
Lipote (Syzygium polycephaloides (C. B. Rob.) Merr.) is a Philippine endemic tree whose berries have a potential for functional food development. This study aimed to investigate the effect of the maturity stage (unripe, half-ripe or fully ripe) and processing (blanching at $90 \pm 5^{\circ} \mathrm{C}$ for 2 mins and steaming at $105 \pm 5^{\circ} \mathrm{C}$ for $5 \mathrm{mins}$ ) on the antioxidant content (total phenolic content (TPC), total flavonoid content (TFC), and total anthocyanin content (TAC)) and antioxidant activity by DPPH, ABTS, and FRAP assays, of lipote flesh and skin (referred herein as flesh) and seeds. Results showed that maturity and processing significantly affect the antioxidant contents and activities of fresh lipote flesh and seeds. Blanched lipote flesh generally had higher TPC, TFC, and antioxidant activities than their unprocessed and steamed counterparts. Blanched unripe flesh had the highest TPC (33.57 $\pm 0.27 \mathrm{mg}$ gallic acid equivalents [GAE]/g) and antioxidant activity measured by ABTS, FRAP, and DPPH assay $(92.31 \pm 1.28 \mathrm{mg}$ Trolox equivalents [TE]/g, $123.83 \pm 0.86 \mathrm{mg} \mathrm{TE} / \mathrm{g}$, and $74.02 \pm 0.33 \mathrm{mg} \mathrm{TE} / \mathrm{g}$, respectively) while blanched fully ripe flesh had the highest TFC (27.98 $\pm 0.08 \mathrm{mg}$ quercetin equivalents $[\mathrm{QE}] / \mathrm{g})$ and TAC $(18.11 \pm 0.11 \mathrm{mg}$ cyanidin-3-glucoside equivalents [C3GE]/g). Lipote seeds, especially unripe ones, have appreciable antioxidant contents and antioxidant activity, even greater than that of lipote flesh. Unprocessed unripe seeds had the greatest TPC, TFC and antioxidant activity by DPPH assay $(123.56 \pm 0.94 \mathrm{mg} \mathrm{GAE} / \mathrm{g}, 111.90 \pm 0.14 \mathrm{mg} \mathrm{QE} / \mathrm{g}$ and $465.89 \pm 1.86 \mathrm{mg} \mathrm{TE} / \mathrm{g}$, respectively). It is recommended that blanching be used as a processing step to increase the antioxidant content of lipote flesh while lipote seeds should be investigated further for nutraceutical applications due to their high antioxidant properties.
\end{abstract}

\section{Introduction}

Today, a rise in global interest in functional foods is evident. Functional foods are those providing health benefits other than basic nutrition. They contain physiologically active components that help reduce chronic disorders like cardiovascular diseases, hypertension, cancer, diabetes, and other diseases. Oxidative stress, the disruption of the balance between reactive oxygen species (ROS) and antioxidant defences in the body, is one condition that has been looked into as it has been associated with obesity, hyperlipidemia, neurological disorders, cancer and chronic obstructive pulmonary disease (Birben et al., 2012).

Antioxidants have been known to mitigate the negative effects of oxidative stress by inactivating ROS, interrupting the radical chain reaction that generates more ROS, and chelating metal ions responsible for the production of ROS, among others (Flora, 2009). The role of dietary antioxidants in alleviating or preventing oxidative stress and related diseases have been continually studied (Duthie et al., 2000; Blomhoff, 2005; Kaliora et al., 2006). Natural pigments and antioxidants are commonly found in most plant materials.

Lipote (Syzygium polycephaloides (C.B. Rob.) Merr.) is a Philippine endemic tree. Its berry is eaten ripe and raw, or juiced and made into wine. Santiago et al. (2007) reported that the antioxidant activity of lipote fruits was comparable to that of Vitamin E. The study also reported that the berry contained $58.85 \mu \mathrm{g}$ catechin 
per gram of fresh sample and exhibited a percent inhibition of linoleic acid peroxidation of $73.7 \%$.

The antioxidant content of berries is affected by maturity and processing (Kalt, 2005; Tiwari and Cummins, 2013). Macheix et al. (1990) reported that the antioxidant activity of dark-coloured fruits like lipote increases with maturity. The same trend was reported by Castillo-Israel et al. (2020) and Sartagoda et al. (2020) for the 'common' and 'kalabaw' variety of bignay (Antidesma bunius (L). Spreng.), respectively. Processing techniques were also reported to have varying effects on the antioxidant contents of fruits and vegetables (Tiwari and Cummins, 2013). Some fruits and vegetables were reported to have increased antioxidant activity when subjected to high temperature processing techniques like boiling, microwaving, and pressure cooking (Kim et al., 2008; Ng and Kuppusamy, 2019). Steaming and blanching were also reported to increase the antioxidant content and activity of bignay (CastilloIsrael et al., 2020; Sartagoda et al., 2021). On the contrary, Chumyam et al. (2013) reported a significant decrease in the antioxidant activity of eggplant when boiled, steamed, and microwaved.

Two common processing steps employed in fruits and vegetables are steaming and blanching. Blanching is a mild heat treatment that primarily aims to inactivate enzymes (Vaclavik and Christian, 2014) while steaming is a cooking method that uses steam generated from boiling water (Lean, 2006).

In a similar vein, the present study aimed to investigate the effect of maturity (unripe, half-ripe, and fully ripe) and processing technique (blanching and steaming) on the antioxidant contents and in vitro antioxidant activity of lipote fruit. Results of this study can be used as evidence for the development of functional foods from lipote.

\section{Materials and methods}

\subsection{Plant material preparation}

Lipote (S. polycephaloides (C. B. Rob) Merr.) fruits were harvested in the province of Laguna, Philippines. The identity of lipote was authenticated by a curator at the Botanical Herbarium, Museum of Natural History, University of the Philippines Los Baños. The collected fruits were washed with water and divided into three maturity stages based on their size and colour (Figure 1).

Each maturity stage was subdivided into three lots. The first lot was subjected to blanching at $90 \pm 5^{\circ} \mathrm{C}$ for 2 mins, the second lot was steamed at $105 \pm 5^{\circ} \mathrm{C}$ for 5 mins, and the last lot was left unprocessed. Blanching was carried out using a water bath while steaming was carried out using a one-layer stainless steel food steamer. All lots were then pulped to separate the seeds from the flesh. The separated flesh and seeds were freeze-dried and ground. The resulting powder was then sieved and stored at room temperature in clean, airtight containers protected from light.

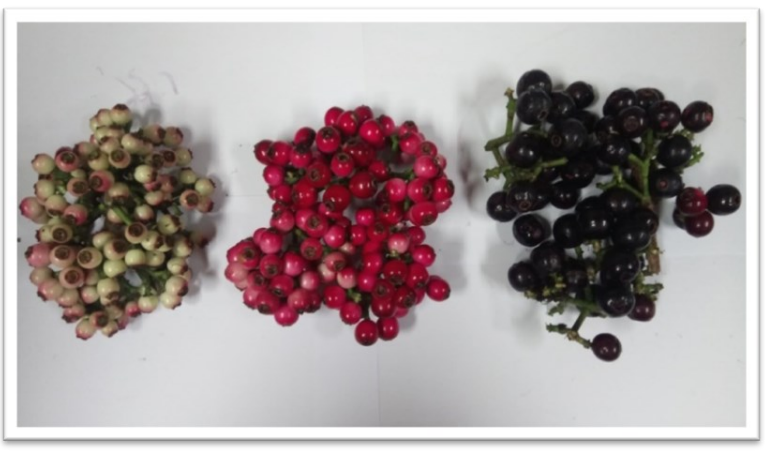

Figure 1. Ripening stages of lipote. From left to right: unripe, half-ripe and fully ripe

\subsection{Sample extraction}

Extraction was done according to the method described by Larrauri et al. (1997) with some modifications. Briefly, $0.5 \mathrm{~g}$ of the freeze-dried sample was extracted with $15 \mathrm{~mL}$ solution of methanol: water: acetic acid (50:50:1, v:v:v) in a shaker for an hour at room temperature. The mixture was then centrifuged for 15 mins. After that, the supernatant was collected and transferred to an amber glass bottle before storing it at 0 $4^{\circ} \mathrm{C}$. Appropriate dilutions of the extracts were done for each analysis.

\subsection{Determination of total phenolic and total flavonoid content}

Quantification of the total phenolic content (TPC) of the samples was done using the Folin-Ciocalteau method described by Waterhouse (2002) with some modifications. A $0.3 \mathrm{~mL}$ portion of the diluted extract was mixed with $1.5 \mathrm{~mL} \mathrm{10 \%}$ Folin-Ciocalteau reagent. The extract was allowed to stand for 5 mins at room temperature. After 5 mins, $1.2 \mathrm{~mL}$ of $4 \% \mathrm{Na}_{2} \mathrm{CO}_{3}$ was added. The solution was vortexed and was allowed to stand for 90 mins. The absorbance readings were measured at $760 \mathrm{~nm}$.

On the other hand, the total flavonoid contents (TFC) of the samples were quantified using the aluminium chloride assay of Luximon-Ramma et al. (2002) with some adjustments. Briefly, a $2.5 \mathrm{~mL}$ portion of the diluted sample was mixed with $0.5 \mathrm{~mL}$ of $10 \% \mathrm{AlCl}_{3}$ solution. The mixture was allowed to stand for $15 \mathrm{mins}$ at room temperature before its absorbance was read at $367.5 \mathrm{~nm}$. 
Methanolic solutions of gallic acid were used as standards for TPC analysis, while methanolic solutions of quercetin were used as standards for TFC analysis. Results were computed as milligrams standard per gram freeze-dried sample.

\subsection{Determination of total anthocyanin content}

The $\mathrm{pH}$ differential method described by Lee et al. (2005), was used to quantify the total anthocyanin content (TAC) of the sample. Potassium chloride buffer $(0.025 \mathrm{M}, \mathrm{pH} 1.0)$ and sodium acetate buffer $(0.40 \mathrm{M}$, $\mathrm{pH}$ 4.5) were used to dilute the extracts to a minimum dilution factor of 5. Dilution factors were chosen in such a way that the absorbance of the sample diluted with the $\mathrm{pH} 1.0$ buffer at $520 \mathrm{~nm}$ is within $0.2-1.2$ absorbance units (AU). The absorbance of the extracts diluted with the $\mathrm{pH} 1.0$ buffer and $\mathrm{pH} 4.5$ buffer were then read at $520 \mathrm{~nm}$ and $700 \mathrm{~nm}$ against a blank of distilled water. After that, the total anthocyanin content (mg cyanidin-3glucoside equivalents/L extract) was computed using Equation (1).

$\mathrm{TAC}(\mathrm{mg} / \mathrm{L})=\mathrm{A} \times \mathrm{MW} \times \mathrm{DF} \times(1000 /(\mathrm{e} \times \mathrm{L}))$

Where $\mathrm{A}$ is the absorbance $=(\mathrm{A} 510 \mathrm{~nm}-\mathrm{A} 700 \mathrm{~nm})$ pH 1.0 - (A510 nm - A700 nm) pH 4.5, MW is the molecular weight of cyanidin-3-glucoside $(449.2 \mathrm{~g} / \mathrm{mol})$, $\mathrm{DF}$ is the dilution factor, e is the extinction coefficient of cyanidin 3 -glucoside $(26,900 \mathrm{~L} / \mathrm{mol} / \mathrm{cm})$ and $\mathrm{L}$ is the cell path length $(1 \mathrm{~cm})$. The computed total anthocyanin content was then converted to milligrams cyanidin-3glucoside equivalents per grams freeze-dried sample.

\subsection{DPPH radical scavenging activity assay}

The DPPH radical scavenging activity assay was adapted based on the method described by Pisoschi and Negulescu (2011). A $1 \mathrm{~mL}$ portion of the diluted sample was mixed with $1 \mathrm{~mL}$ absolute methanol and $1 \mathrm{~mL}$ of freshly prepared DPPH solution. Methanolic solutions of Trolox were used as standards. The absorbance was then read at $517 \mathrm{~nm}$ against a blank of absolute methanol. Results were expressed as milligram Trolox equivalents per gram freeze-dried sample.

\subsection{ABTS assay}

The ABTS or TEAC assay was done according to the method described by Tomasina et al. (2012) with some adjustments. The ABTS radical cation was prepared by reacting a $0.25 \mathrm{mM}$ ABTS stock solution with $3.45 \mathrm{mM}$ potassium persulfate, allowing the mixture to stand in the dark for $12-16$ hours at $4^{\circ} \mathrm{C}$. After this, the ABTS radical cation solution was diluted with methanol until an absorbance of $0.70 \pm 5$ at $734 \mathrm{~nm}$ was achieved. Then, $2.7 \mathrm{~mL}$ of ABTS radical cation solution was added to $0.3 \mathrm{~mL}$ of the diluted sample. The mixture was allowed to stand at room temperature in the dark for 15 mins. Methanolic solutions of Trolox were used as standards. The absorbance of the solution was measured at $734 \mathrm{~nm}$. Results were expressed as $\mathrm{mg}$ Trolox equivalents per $\mathrm{g}$ sample.

\subsection{Ferric reducing antioxidant power (FRAP) assay}

The FRAP assay of the samples was done using a modified method described by Tomasina et al. (2012). Briefly, $2.7 \mathrm{~mL}$ of freshly prepared FRAP reagent (300 $\mathrm{mM}$ acetate buffer (pH 3.6):10 mM TPTZ $(10 \mathrm{mM}): 20$ $\mathrm{mM} \mathrm{FeCl}_{3}$ solution; $(10: 1: 1, \mathrm{v}: \mathrm{v}: \mathrm{v})$ was added to $0.3 \mathrm{~mL}$ of the diluted sample. The reaction mixture was incubated at $37^{\circ} \mathrm{C}$ for 5 mins before its absorbance was read at $620 \mathrm{~nm}$. Methanolic solutions of Trolox were used as standards. Results were reported as mg Trolox equivalents per $g$ sample.

\subsection{Statistical analysis}

The experiments were arranged in a completely randomized design with values presented as mean \pm SEM computed from three values. The influence of the maturity stage and processing treatment on TFC, TPC, and TAC and antioxidant activities (ABTS, DPPH, and FRAP) of fruit seeds and flesh were analysed using a two -factor factorial ANOVA. Differences between mean values were assessed by Tukey's Honest Significant Difference (HSD) test. All the statistical tests were performed at the significance level of 0.05 using Minitab 19.0 for Windows (Minitab, 2019).

\section{Results}

3.1 Effect of maturity and processing method on the TPC, TFC, and TAC of lipote flesh

Maturity and processing, as well as their interaction, have significant effects on the TPC, TFC, and TAC of lipote flesh $(\mathrm{p} \leq 0.05)$. Blanched unripe lipote flesh had the highest total phenolic content $(33.57 \pm 0.27 \mathrm{mg}$ GAE/ g) while blanched fully ripe lipote flesh had the highest total flavonoid content and total anthocyanin content $(27.98 \pm 0.08 \mathrm{mg} \mathrm{QE} / \mathrm{g}$ and $18.11 \pm 0.11 \mathrm{mg} \mathrm{C} 3 \mathrm{GE} / \mathrm{g}$, respectively). Generally, blanched flesh had higher TPC and TFC than both their steamed and unprocessed counterparts. Moreover, steamed flesh generally had higher TPC and TFC than the unprocessed counterparts. In addition, the thermally processed seeds had greater TAC values than their unprocessed counterparts (Figure 2, Table 1).

For fully-ripe lipote flesh, blanching caused a $64.8 \%$ increase in TPC and a $38.6 \%$ increase in TFC while steaming caused a $60.2 \%$ increase in TPC and a $30.9 \%$ increase in TFC. For half-ripe lipote flesh, blanching 

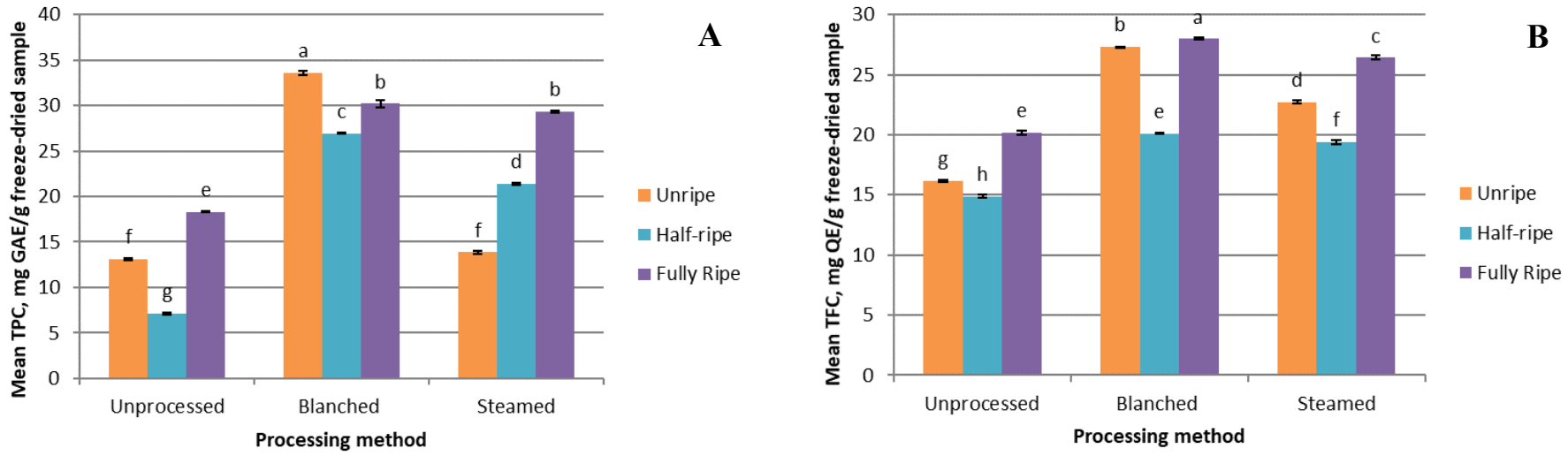

Figure 2. Total phenolic content (A) and total flavonoid content (B) of lipote flesh as affected by maturity and processing method before freeze-drying $($ mean \pm SEM). Bars with different lowercase letters denote a significant difference $(p \leq 0.05$; Tukey's test)

caused a $277 \%$ increase in TPC and $35.4 \%$ increase in TFC while steaming caused a $199 \%$ increase in TPC and $30.6 \%$ increase in TFC. For unripe lipote flesh, blanching caused a $156 \%$ increase in TPC and $69.1 \%$ increase in TFC while steaming caused a $5.73 \%$ increase in TPC and a $40.7 \%$ increase in TFC (Figure 2).

Table 1. Total anthocyanin content of lipote flesh

\begin{tabular}{ccc}
\hline $\begin{array}{c}\text { Processing } \\
\text { method }\end{array}$ & $\begin{array}{c}\text { Maturity } \\
\text { stage }\end{array}$ & $\begin{array}{c}\text { Total anthocyanin content, mg } \\
\text { cyanidin-3-glucoside equivalents/ } \\
\text { g freeze-dried sample }\end{array}$ \\
\hline Unprocessed & Unripe & $\mathrm{ND}$ \\
& Half-ripe & $0.28 \pm 0.02^{\mathrm{e}}$ \\
& Fully ripe & $8.02 \pm 0.43^{\mathrm{c}}$ \\
\hline Blanched & Unripe & $\mathrm{ND}$ \\
& Half-ripe & $0.89 \pm 0.00^{\mathrm{e}}$ \\
& Fully ripe & $18.11 \pm 0.11^{\mathrm{a}}$ \\
\hline Steamed & Unripe & $\mathrm{ND}$ \\
& Half-ripe & $1.78 \pm 0.00^{\mathrm{d}}$ \\
& Fully ripe & $13.25 \pm 0.01^{\mathrm{b}}$ \\
\hline
\end{tabular}

Values are expressed as mean $\pm \mathrm{SEM}, \mathrm{n}=3$. Values with different superscript are significantly different (Tukey's test, $p$ $\leq 0.05)$. ND, not detected.

TAC of lipote flesh increases as the fruit matures regardless of processing method before freeze-drying (Table 1). On the other hand, the influence of maturity in TPC is variable. For unprocessed flesh, TPC decreases from the unripe stage to the half-ripe stage and then increases markedly upon reaching the fully ripe stage. For blanched flesh, TPC decreases from the unripe stage to the half-ripe stage and decreases marginally upon reaching the fully ripe stage. For steamed flesh, TPC increases as the fruit matures. Finally, TFC is usually greatest at the fully ripe stage and the least at the halfripe stage for all processing methods before freezedrying (Figure 2).
3.2 Effect of maturity and processing method on the antioxidant activity (ABTS, DPPH, and FRAP) of lipote flesh

Maturity and processing, as well as their interaction, have significant effects on the antioxidant activities measured by ABTS, DPPH, and FRAP assays of lipote flesh $(p<0.05)$. Blanched unripe lipote flesh had the highest antioxidant activity measured by ABTS, FRAP, and DPPH assay $(92.31 \pm 1.28 \mathrm{mg} \mathrm{TE} / \mathrm{g}, 123.83 \pm 0.86 \mathrm{mg}$ $\mathrm{TE} / \mathrm{g}$, and $74.02 \pm 0.33 \mathrm{mg} \mathrm{TE} / \mathrm{g}$, respectively). Blanched flesh generally had higher antioxidant activities measured by ABTS, FRAP, and DPPH assays than both their steamed and unprocessed counterparts. Moreover, steamed flesh had higher antioxidant activities measured by ABTS, FRAP, and DPPH assays than their unprocessed counterparts (Figure 3).

For fully ripe lipote flesh, blanching caused an $81.4 \%, 85.9 \%$ and $57.4 \%$ increase in antioxidant activity measured by ABTS, FRAP and DPPH assays respectively while steaming caused a $77.7 \%, 107 \%$ and $60.4 \%$ increase in antioxidant activity by the aforementioned assays, respectively. For half-ripe lipote flesh, blanching caused a $192 \%, 365 \%$, and $118 \%$ increase in antioxidant activity measured by ABTS, FRAP and DPPH assays respectively while steaming caused a $176 \%, 249 \%$, and $74.4 \%$ increase in antioxidant activity by the aforementioned assays respectively. For unripe lipote flesh, blanching caused a $66.0 \%, 185 \%$ and $133 \%$ increase in antioxidant activity measured by ABTS, FRAP and DPPH assays respectively while steaming caused a $13.6 \%, 4.29 \%$ and $0.178 \%$ increase in antioxidant activity by the aforementioned assays respectively (Figure 3).

The effect of maturity on the antioxidant activities of the lipote flesh is variable. For antioxidant activity measured by the ABTS assay, antioxidant activity is greatest at the unripe stage and then markedly decreases upon reaching the half-ripe stage. For antioxidant 


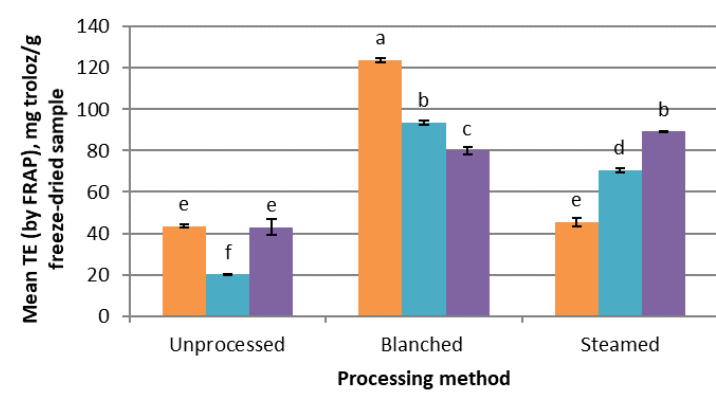

A

Unripe

- Half-ripe

- Fully Ripe

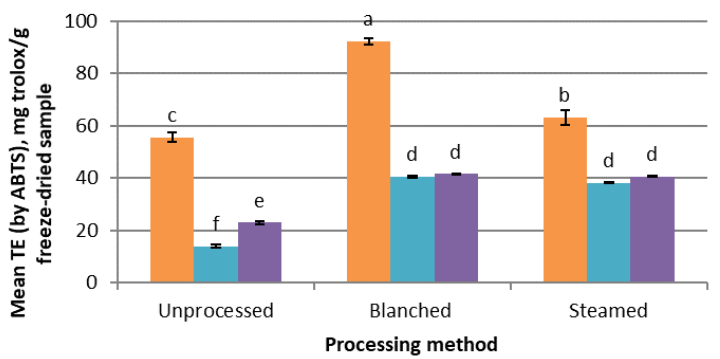

B

Unripe

- Half-ripe

- Fully Ripe

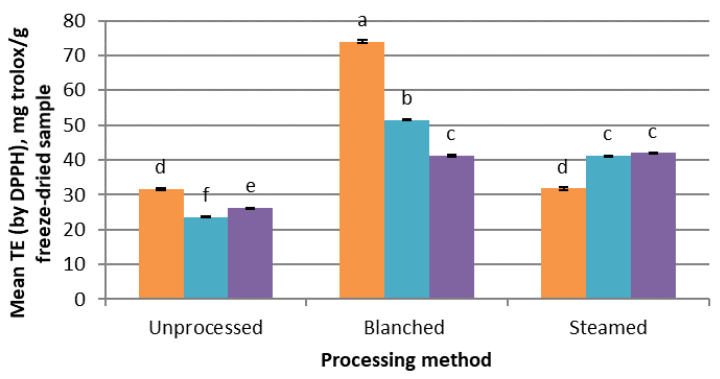

C

In Unripe

- Half-ripe

- Fully Ripe

Figure 3. Antioxidant activity of lipote flesh measured by FRAP (A), ABTS (B), and DPPH (C) assays expressed as mg. Trolox equivalents per $\mathrm{g}$ freeze-dried sample as affected by maturity and processing method before freeze-drying (mean \pm SEM). Bars with different lowercase letters denote a significant difference $(\mathrm{p} \leq 0.05$; Tukey's test).

activity measured by FRAP assay, blanched flesh decreases in antioxidant activity as the fruit matures, steamed flesh increases in antioxidant activity as the fruit matures, and finally, unprocessed flesh decreases in antioxidant activity upon reaching the half-ripe stage before returning to the same level upon reaching the fully ripe stage. For antioxidant activity measured by the DPPH assay, blanched flesh still decreases in antioxidant activity as the fruit matures. However, steamed flesh increases antioxidant activity by DPPH assay upon reaching the half-ripe stage and stays at that level upon reaching the fully ripe stage. Moreover, unprocessed flesh decreases in antioxidant activity measured by DPPH upon reaching the half-ripe stage and then increases slightly upon reaching the fully ripe stage (Figure 3).

3.3 Effect of maturity and processing method on the TPC, TFC, and TAC of lipote seeds

Maturity and processing, as well as their interaction, have significant effects on the TPC, TFC, and TAC of lipote seeds $(p \leq 0.05)$. The TPC and TFC of lipote seeds generally decrease as the fruit matures. Moreover, unprocessed unripe seeds had the greatest TPC and TFC (123.56 $\pm 0.94 \mathrm{mg}$ GAE/g and $111.90 \pm 0.14 \mathrm{mg} \mathrm{QE} / \mathrm{g}$, respectively) among all the other samples (Figure 4).

Unprocessed unripe seeds had a $42.3 \%$ decrease in TPC upon reaching the half-ripe stage and a $77.0 \%$ decrease in the same parameter upon reaching the fully ripe stage. Unprocessed unripe seeds also had a $44.9 \%$ decrease in TFC upon reaching the half-ripe stage and a $69.0 \%$ decrease in the same parameter upon reaching the fully ripe stage (Figure 4).

Blanched unripe seeds had a $17.1 \%$ decrease in TPC upon reaching the half-ripe stage and a $53.9 \%$ decrease in the same parameter upon reaching the fully ripe stage. Blanched unripe lipote seeds also had a $1.24 \%$ decrease in TFC upon reaching the half-ripe stage and a decrease in the same parameter upon reaching the fully ripe stage (Figure 4).

Steamed unripe seeds had a $27.9 \%$ decrease in TPC stage and a $43.8 \%$ decrease in the same parameter upon reaching the fully ripe stage. Steamed unripe seeds also had a $28.7 \%$ decrease in TFC upon reaching the half-ripe stage and a $47.4 \%$ decrease in the same parameter upon reaching the fully ripe stage (Figure 4).
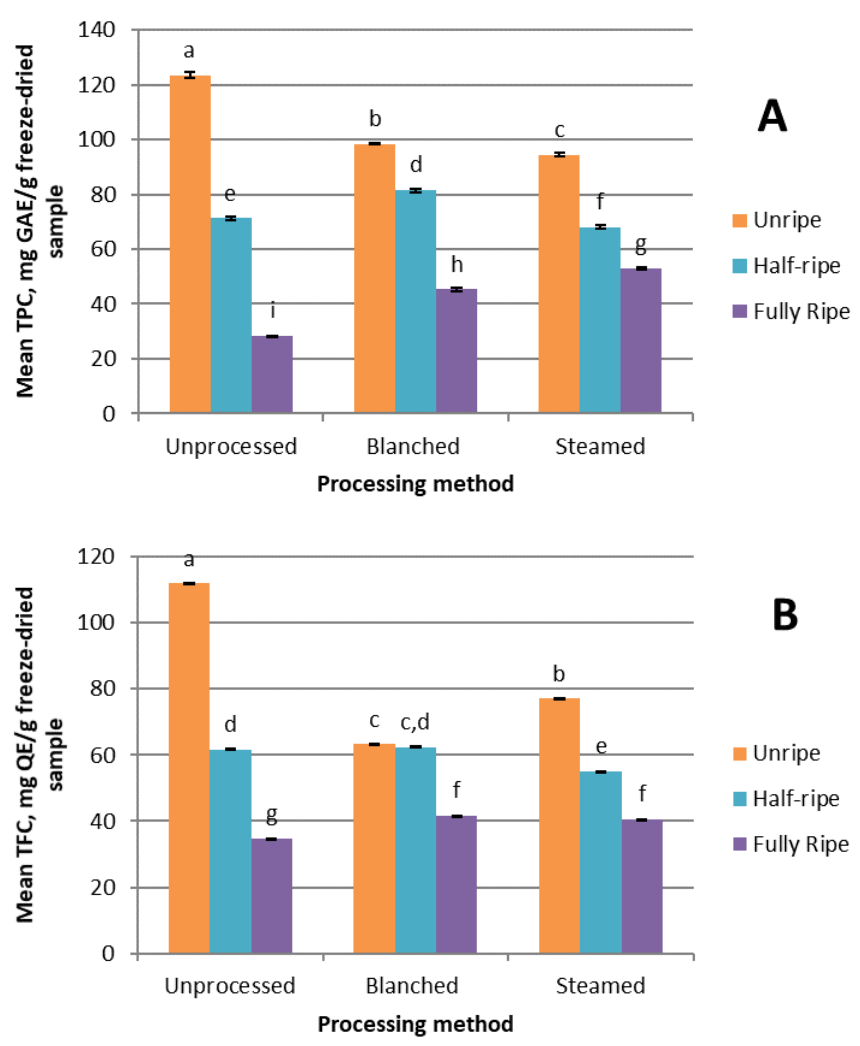

Figure 4. Total phenolic content (A) and total flavonoid content (B) of lipote seeds as affected by maturity and processing method before freeze-drying (mean \pm SEM). Bars with different lowercase letters denote a significant difference ( $\mathrm{p} \leq 0.05$; Tukey's test) 
The TAC of lipote seeds ranged from $0.22 \pm 0.01 \mathrm{mg}$ cyanidin-3-glucoside/g to $1.13 \pm 0.00 \mathrm{mg}$ cyaniding $/ \mathrm{g}$. Processing generally increases anthocyanin content of the seeds. Both steamed and blanched seeds have greater TAC than their unprocessed counterparts (Table 2).

Table 2. Total anthocyanin content of lipote seeds

\begin{tabular}{ccc}
\hline $\begin{array}{c}\text { Processing } \\
\text { method }\end{array}$ & $\begin{array}{c}\text { Maturity } \\
\text { stage }\end{array}$ & $\begin{array}{c}\text { Total anthocyanin content, mg } \\
\text { cyanidin-3-glucoside equivalents/ } \\
\text { g freeze-dried sample }\end{array}$ \\
\hline Unprocessed & Unripe & ND \\
& Half-ripe & ND \\
& Fully ripe & $0.08 \pm 0.00^{\mathrm{e}}$ \\
\hline Blanched & Unripe & $\mathrm{ND}$ \\
& Half-ripe & $0.22 \pm 0.01^{\mathrm{c}}$ \\
& Fully ripe & $0.40 \pm 0.00^{\mathrm{b}}$ \\
\hline Steamed & Unripe & $\mathrm{ND}$ \\
& Half-ripe & $0.13 \pm 0.00^{\mathrm{d}}$ \\
& Fully ripe & $0.78 \pm 0.00^{\mathrm{a}}$ \\
\hline
\end{tabular}

Values are expressed as mean \pm SEM, $\mathrm{n}=3$. Values with different superscript are significantly different (Tukey's test, $p$ $\leq 0.05)$. ND, not detected.

\subsection{Effect of maturity and processing method on the antioxidant activity (ABTS, DPPH, and FRAP) of lipote seeds}

Maturity and processing, as well as their interaction, have significant effects on the antioxidant activities measured by ABTS, DPPH, and FRAP assays of lipote flesh $(\mathrm{p} \leq 0.05)$.

The antioxidant activity of unripe seeds is generally greater than that of their half-ripe and unripe counterparts. For unprocessed seeds, antioxidant activities measured by ABTS, FRAP and DPPH assays decreased by $51.9 \%, 34.3 \%$, and $50.2 \%$ upon reaching the half-ripe stage and $68.1 \%, 78.8 \%$ and $80.5 \%$ upon reaching the fully ripe stage. On the other hand, for blanched seeds, antioxidant activities measured by ABTS, FRAP and DPPH assays decreased by $15.1 \%$, $23.1 \%$ and $27.8 \%$ upon reaching the half-ripe stage and $61.2 \%, 55.7 \%$ and $57.5 \%$ upon reaching the fully ripe stage. Finally, for steamed seeds, antioxidant activities measured by ABTS, FRAP and DPPH assays decreased by $56.4 \%, 36.5 \%$, and $41.3 \%$ upon reaching the half-ripe stage and $58.1 \%, 48.0 \%$ and $35.2 \%$ upon reaching the fully ripe stage.

Unprocessed unripe seeds and steamed unripe seeds had the greatest antioxidant activity measured by ABTS assay $(227.93 \pm 12.21 \mathrm{mg}$ TE/g and $270.17 \pm 2.24 \mathrm{mg}$ TE/ g). On the other hand, unprocessed unripe seeds and blanched unripe seeds had the greatest antioxidant activity as measured by FRAP assay $(479.10 \pm 0.82 \mathrm{mg}$ $\mathrm{TE} / \mathrm{g}$ and $480.80 \pm 1.91 \mathrm{mg} \mathrm{TE} / \mathrm{g}$, respectively). Finally, only unprocessed unripe seeds had the greatest antioxidant activity as measured by the DPPH assay (465.89 $\pm 1.86 \mathrm{mg} \mathrm{TE} / \mathrm{g}$ ) (Figure 5).
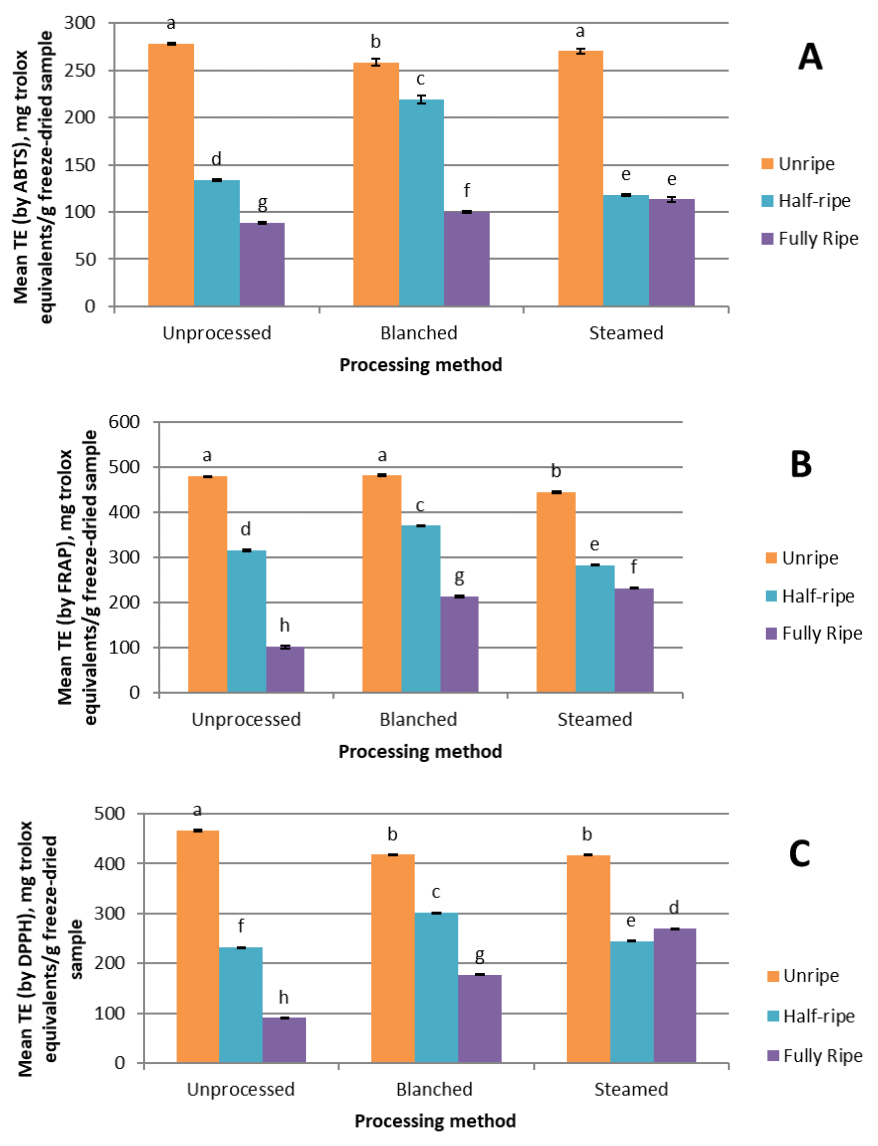

Figure 5. Antioxidant activity of lipote seeds measured by ABTS (A), FRAP (B), and DPPH (C) assays (top to bottom) expressed as mg Trolox equivalents per $\mathrm{g}$ freeze-dried sample as affected by maturity and processing method before freezedrying (mean \pm SEM). Bars with different lowercase letters denote a significant difference $(\mathrm{p} \leq 0.05$; Tukey's test $)$

\section{Discussion}

This study was undertaken to investigate the effect of maturity stage and processing (specifically blanching and steaming) on the antioxidant contents and antioxidant activities of lipote flesh and seeds for the purpose of developing functional foods.

The total phenolics, total flavonoids, and total anthocyanin content of lipote flesh as well as their antioxidant activities measured by ABTS, DPPH, and FRAP were all significantly associated with both maturity stage and processing method $(\mathrm{p} \leq 0.05)$. Moreover, on all these parameters, the interaction between the maturity stage and processing method was significant. This was also observed in the study of Sartagoda et al. (2021) on bignay 'kalabaw' and Castillo -Israel et al. (2020) on bignay 'common.'

HPLC analysis by Chirinos et al. (2010) on Peruvian camu-camu and by Hammouda et al. (2013) on Tunisian dates showed that the concentration of phenolic 
compounds changes during ripening. These changes may account for the effect of maturity in the antioxidant content and antioxidant activity of the samples. These may also account for the interaction between maturity and processing, as the compounds that changed in concentration may have different sensitivity to heat treatment. Still, this needs further investigation.

The total phenolic content and total antioxidant activities of blanched flesh are generally greater than that of the unprocessed flesh. This was also observed in the study of Sartagoda et al. (2021) on bignay 'kalabaw' and Castillo-Israel et al. (2020) on bignay 'common.' Increases in the total phenolic content and antiradical potential by DPPH assay due to blanching pre-treatment prior to freeze-drying was also observed in the study of Franceschinis et al. (2015) on cherry discs. Results of this study showed that blanching prior to air-drying and freeze-drying produced a product that has a greater TPC and ARP by DPPH assay than those that were not subjected to treatment and those that were subjected to sugar infusion prior to the two aforementioned drying techniques. The authors of this study speculated that this could be due to the inactivation of polyphenol oxidase during blanching, preventing the breakdown of some polyphenols in the fruit. In a similar study by Sablani et al. (2011), steam blanching prior to air drying of all but one of the four types of red raspberries and four types of blueberries studied resulted in a greater phenolic content of the product as compared to those air-dried without blanching as a pre-treatment. However, results on the effect of steam blanching on antioxidant activity are more variable, with some types having greater antioxidant activity when blanching was used a pretreatment prior to air-drying, some types having lesser antioxidant activity and some types having no significant differences in antioxidant activities. Blanching was also found to increase the ellagatannin content of blackberries for juice processing (Hager et al., 2010). In addition to the inactivation of enzymes, blanching can also break down the cell matrix, which can subsequently increase the extraction of phenolics (Nayak et al., 2015). Blanched unripe lipote flesh had the highest antioxidant activity measured by DPPH, ABTS, and FRAP. This finding coincides with it having the greatest phenolic content out of all the other samples.

Total anthocyanin content markedly increases during the ripening of the flesh. This is consistent with the study of Yang et al. (2016) on mulberries and Seraglio et al. (2018) on Myrciaria cauliflora, Myrcianthes pungens and Syzygium cumuni. This also coincides with the study of Sartagoda et al. (2021) on bignay 'kalabaw' and Castillo-Israel et al. (2020) on bignay 'common.' This correlates with the colour development of the fruit.
Moreover, in the study of Sablani et al. (2011), steam blanching prior to air drying decreases the degradation of anthocyanins. This may coincide with the greater TAC content observed in the thermally processed samples as compared to the unprocessed samples.

The total phenolics, total flavonoids, and total anthocyanin content of lipote seeds as well as their antioxidant activities measured by ABTS, DPPH, and FRAP were also all significantly associated with both maturity stage and processing method $(p \leq 0.05)$. The interaction between the maturity stage and processing method in all these parameters was also significant. This was also observed in the study of Sartagoda et al. (2021) on bignay 'kalabaw' and Castillo-Israel et al. (2020) on bignay 'common.'

Unripe lipote seeds generally had greater TPC, TFC, and antioxidant activities than their half-ripe and fully ripe counterparts. This is in contrast to the study of Sartagoda et al. (2020) on bignay 'kalabaw' and Castillo -Israel et al. (2020) on bignay 'common' which reported that fully ripe seeds of both berries generally had the greatest TPC, TFC and antioxidant activities by ABTS, FRAP and DPPH than their half-ripe and fully ripe counterparts. In the study of Moulehi et al. (2012), the TPC, TFC, and antioxidant activity measured by DPPH of mandarin orange and bitter orange were also affected by maturity. The effects of maturity on TPC varied in the two types of seeds. Like those of the lipote seeds, the TPC of the mandarin orange seeds in this study also decreased during ripening. However, in both the mandarin orange and bitter orange seeds, the antioxidant activity measured by DPPH increased during ripening. Differences in the antioxidant activities exhibited by the orange seeds and lipote seeds may be due to the nature of the fruit themselves. Total phenolic contents of seeds of Syzygium cumini was also reported to decrease as the fruit matures (Kheaw-on et al., 2008).

Little to no anthocyanins was detected in the seeds of lipote. This coincides with the study of Aqil et al. (2012) and Kheaw-on et al. (2008) which reported that anthocyanins were not detected in the seeds of Syzygium cumini. Moreover, greater TAC of the seeds of the thermally processed fruits may be due to the migration of anthocyanins from the flesh to the seeds during processing.

The TPC, TFC, and antioxidant activities measured by ABTS, DPPH, and FRAP of lipote seeds were considerably higher than that of the lipote flesh. Seeds of Syzygium cumini, were also reported to contain higher phenolic content and reducing capacity by FRAP assay than its flesh (Kheaw-on et al., 2008). Seeds also contained higher phenolic content than the skin and pulp 
of Muscadine grapes (Vitis rotundifolia), according to the study of Sandhu and Gu (2010).

Possible implications of these findings for functional food development are as follows. First, blanching can be used to increase the phenolic content and antioxidant activity of lipote flesh. Second, lipote seeds, especially unripe ones, have good potential for nutraceutical applications due to their appreciable antioxidant contents.

\section{Conclusion}

The maturity stage and processing method both significantly affect the total phenolic content, total flavonoid content, total anthocyanin content, and antioxidant activities of lipote seeds and flesh. Moreover, the interaction of maturity and processing on all these parameters was statistically significant.

Generally, blanched flesh was found to have greater total flavonoid content, total anthocyanin content, and antioxidant activities than their unprocessed and steamed counterparts. Therefore, for functional food development, blanching can be used as a processing step to increase the antioxidant content of lipote flesh.

Seeds have appreciable total flavonoid content, total anthocyanin content, and antioxidant activities, even greater than that of lipote flesh. Moreover, unripe seeds generally have greater total flavonoid content, total anthocyanin content, and antioxidant activities than their half-ripe and fully ripe counterparts. For future functional food development, seeds can be investigated further for nutraceutical applications.

\section{Acknowledgement}

The authors would like to acknowledge the funding from the Department of Science and Technology Philippine Council for Health Research and Development (DOST- PCHRD) program on Philippine Indigenous Berries (BERRYPINOY).

\section{References}

Aqil, F., Gupta, A., Munagala, R., Jeyabalan, J., Kausar, H., Sharma, R.J., Singh, I.P. and Gupta, R.C. (2012). Antioxidant and antiproliferative activities of anthocyanin/ellagitannin-enriched extracts from Syzygium cumini L. (Jamun, the Indian Blackberry). Nutrition and Cancer, 64(3), 428-438. https://doi.org/10.1080/01635581.2012.657766

Birben, E., Sahiner, U.M., Sackesen, C., Erzurum, S. and Kalayci, O. (2012). Oxidative stress and antioxidant defense. The World Allergy Organization Journal, 5
(1), 9-19.

Blomhoff, R. (2005). Dietary antioxidants and cardiovascular disease. Current Opinion in Lipidology, 16(1), 47-54. https:// doi.org/10.1097/00041433-200502000-00009

Castillo- Israel, K.A.T., Sartagoda, K.J.D., Ilano, M.C.R., Flandez, L.E.L., Compendio, M.C.M. and Morales, D.B. (2020). Antioxidant Properties of Philippine Bignay (Antidesma bunius (Linn.) Spreng cv. 'Common') Flesh and Seeds as affected by Fruit Maturity and Heat Treatment. Food Research, 4(6), 1980 - 1987. https://doi.org/10.26656/fr.2017.4 (6). 215

Chirinos, R., Galarza, J., Betalleluz-Pallardel, I., Pedreschi, R. and Campos, D. (2010). Antioxidant compounds and antioxidant capacity of Peruvian camu camu (Myrciaria dubia (HBK) McVaugh) fruit at different maturity stages. Food Chemistry, 120(4), 1019-1024.

https://doi.org/10.1016/ j.foodchem.2009.11.041

Chumyam, A., Whangchai, K., Jungklang, J., Faiyue, B. and Saengnil, K. (2013). Effects of heat treatments on antioxidant capacity and total phenolic content of four cultivars of purple skin eggplants. Science Asia, 39, 246-251. https://doi.org/10.2306/scienceasia1513 $-1874.2013 .39 .246$

Duthie, G.G., Duthie, S.J. and Kyle, J.A. (2000). Plant polyphenols in cancer and heart disease: implications as nutritional antioxidants. Nutrition Research Reviews, 13(1), 79-106. https:// doi.org/10.1079/095442200108729016

Flora, S.J. (2009). Structural, chemical and biological aspects of antioxidants for strategies against metal and metalloid exposure. Oxidative Medicine and Cellular Longevity, 2(4), 191-206. https:// doi.org/10.4161/oxim.2.4.9112

Franceschinis, L., Sette, P., Schebor, C. and Salvatori, D. (2015). Color and bioactive compounds characteristics on dehydrated sweet cherry products. Food and Bioprocess Technology, 8(8), 1716-1729. https://doi.org/10.1007/s11947-015-1533-9

Hager, T.J., Howard, L.R. and Prior, R.L. (2010). Processing and storage effects on the ellagitannin composition of processed blackberry products. Journal of Agricultural and Food Chemistry, 58(22), 11749-11754. https:// doi.org/10.1021/jf102964b

Hammouda, H., Chérif, J.K., Trabelsi-Ayadi, M., Baron, A. and Guyot, S. (2013). Detailed polyphenol and tannin composition and its variability in Tunisian dates (Phoenix dactylifera L.) at different maturity stages. Journal of Agricultural and Food Chemistry, 
61(13), 3252-3263. https://doi.org/10.1021/jf304614j

Kaliora, A.C., Dedoussis, G.V.Z. and Schmidt, H. (2006). Dietary antioxidants in preventing atherogenesis. Atherosclerosis, 187(1), 1-17. https:// doi.org/10.1016/j.atherosclerosis.2005.11.001

Kalt, W. (2005). Effects of production and processing factors on major fruit and vegetable antioxidants. Journal of Food Science, 70(1), 11-19. https:// doi.org/10.1111/j.1365-2621.2005.tb09053.x

Kheaw-on, N., Chaisuksant, R. and Suntornwat, O. (2008, August). Antioxidant capacity of flesh and seed from Syzygium cumini fruits. Acta Horticulturae, 837, 73-78. https://doi.org/10.17660/ ActaHortic.2009.837.7

Kim, H.Y., Woo, K.S., Hwang, I.G., Lee, Y.R. and Jeong, H.S. (2008). Effects of heat treatments on the antioxidant activities of fruits and vegetables. Korean Journal of Food Science and Technology, 40 (2), 166-170.

Larrauri, J.A., Rupérez, P. and Saura-Calixto, F. (1997). Effect of drying temperature on the stability of polyphenols and antioxidant activity of red grape pomace peels. Journal of Agricultural and Food Chemistry, 45(4), 1390-1393. https:// doi.org/10.1021/jf960282f

Lean, M.E.J. (2006). Fox and Cameron's Food Science, Nutrition and Health. 7th ed. Boca Raton, Florida, USA: Taylor and Francis Group, LLC. https:// doi.org/10.1201/b13442

Lee, J., Durst, R.W. and Wrolstad, R.E. (2005). Determination of total monomeric anthocyanin pigment content of fruit juices, beverages, natural colorants, and wines by the $\mathrm{pH}$ differential method: collaborative study. Journal of AOAC international, 88(5), 1269-1278. https://doi.org/10.1093/ jaoac/88.5.1269

Luximon-Ramma, A., Bahorun, T., Soobrattee, M.A. and Aruoma, O.I. (2002). Antioxidant activities of phenolic, proanthocyanidin, and flavonoid components in extracts of Cassia fistula. Journal of Agricultural and Food Chemistry, 50(18), 50425047. https://doi.org/10.1021/jf0201172

Macheix, J-J., Fleuriet, A. and Billot, J. (Eds.)(1990). Phenolic composition of individual fruits. Fruit Phenolics, p. 105-148. USA: CRC Press.

Moulehi, I., Bourgou, S., Ourghemmi, I. and Tounsi, M.S. (2012). Variety and ripening impact on phenolic composition and antioxidant activity of mandarin (Citrus reticulate Blanco) and bitter orange (Citrus aurantium L.) seeds extracts. Industrial Crops and Products, 39, 74-80. https:// doi.org/10.1016/j.indcrop.2012.02.013
Nayak, B., Liu, R.H. and Tang, J. (2015). Effect of processing on phenolic antioxidants of fruits, vegetables, and grains-a review. Critical Reviews in Food Science and Nutrition, 55(7), 887-918. https://doi.org/10.1080/10408398.2011.654142

Ng, Z.X. and Kuppusamy, U.R. (2019). Effects of different heat treatments on the antioxidant activity and ascorbic acid content of bitter melon, Momordica charantia. Brazilian Journal of Food Technology, 22, e2018283. https:// doi.org/10.1590/1981-6723.28318

Pisoschi, A.M. and Negulescu, G.P. (2011). Methods for total antioxidant activity determination: a review. Biochemistry and Analytical Biochemistry, 1(1), 106.

Sablani, S.S., Andrews, P.K., Davies, N.M., Walters, T., Saez, H. and Bastarrachea, L. (2011). Effects of air and freeze drying on phytochemical content of conventional and organic berries. Drying Technology, 29(2), 205-216. https:// doi.org/10.1080/07373937.2010.483047

Sandhu, A.K. and Gu, L. (2010). Antioxidant capacity, phenolic content, and profiling of phenolic compounds in the seeds, skin, and pulp of Vitis rotundifolia (muscadine grapes) as determined by HPLC-DAD-ESI-MS ${ }^{\mathrm{n}}$. Journal of Agricultural and Food Chemistry, 58(8), 4681-4692. https:// doi.org/10.1021/jf904211q

Santiago, D.M.O., Garcia, V.V., Dizon, E.I. and Merca, F.E. (2007). Antioxidant Activities, Flavonol and Flavanol Content of Selected Southeast Asian Indigenous Fruits. Philippine Agricultural Scientist, 90(2), 123-130.

Seraglio, S.K.T., Schulz, M., Nehring, P., Della Betta, F., Valese, A.C., Daguer, H., Gonzaga, L.V., Fett, R. and Costa, A.C.O. (2018). Nutritional and bioactive potential of Myrtaceae fruits during ripening. Food Chemistry, 239, 649-656. https://doi.org/10.1016/ j.foodchem.2017.06.118

Sartagoda, K.J., Ilano, M.C., Flandez, L.E. and CastilloIsrael, K.A. (2021). Evaluation of the Antioxidant Activity of Bignay (Antidesma bunius (Linn.) Spreng var. Kalabaw) Flesh and Seeds as affected by Maturity and Processing Method. Chang Mai University Journal of Natural Sciences, 20(2), e2021042.

CMUJNS.2021.042

Tiwari, U. and Cummins, E. (2013). Factors influencing levels of phytochemicals in selected fruit and vegetables during pre-and post-harvest food processing operations. Food Research International, 50(2), 497-506. https://doi.org/10.1016/ j.foodres.2011.09.007 
Tomasina, F., Carabio, C., Celano L. and Thomson, L. (2012). Analysis of two methods to evaluate antioxidants. Biochemistry and Molecular Biology Education, 40(4), 266-270. https://doi.org/10.1002/ bmb.20617

Vaclavik, V.A. and Christian, E.W. (2014). Essentials of Food Science, p. 326. New York, NY: Springer Science+Business Media. https:// doi.org/10.1007/978-1-4614-9138-5

Waterhouse, A.L. (2002). Determination of total phenolics. Current Protocols in Food Analytical Chemistry, 6(1), I1.1.1 - I1.1.8. https:// doi.org/10.1002/0471142913.faa0101s06

Yang, J., Liu, X., Zhang, X., Jin, Q. and Li, J. (2016). Phenolic profiles, antioxidant activities, and neuroprotective properties of mulberry (Morus atropurpurea Roxb.) fruit extracts from different ripening stages. Journal of Food Science, 81(10), C2439-C2446. https://doi.org/10.1111/17503841.13426 